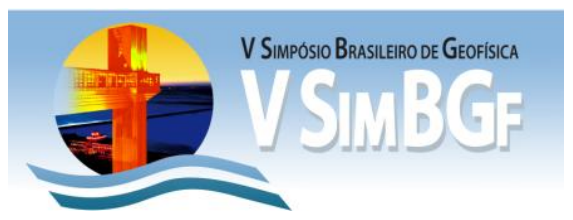

\title{
Estimativa de parâmetros do perfil NMR em reservatórios carbonáticos da Bacia de Campos utilizando perfis geofísicos de poço e técnicas de inteligência artificial
}

\author{
Briones, V. \& Carrasquilla, A., LENEP/UENF, MACAÉ - RJ
}

Copyright 2012, SBGf - Sociedade Brasileira de Geofísica

Este texto foi preparado para a apresentação no V Simpósio Brasileiro de Geofísica, Salvador, 27 a 29 de novembro de 2012 . Seu conteúdo foi revisado pelo Comitê Salvador, 27 a 29 de novembro de 2012. Seu conteúdo foi revisado pelo Comitê seus associados. É proibida a reprodução total ou parcial deste material para propósitos comerciais sem prévia autorização da SBGf.

\section{Resumo}

The Nuclear Magnetic Resonance (NMR) well log provides useful information for the petrophysical study of oil reservoirs. By the processing and the interpretation of NMR log, it is possible to obtain parameters such as total porosity, effective porosity, free fluid porosity, permeability estimative and water, gas and oil saturations, all independent of lithology. In other words, this tool is able to predict the petrophysical parameters of the formations more effectively than conventional logging tools, allowing a better interpretation and leading to more accurate results. The present study evaluated the reliability of the use of intelligent systems with the goal to simulate parameters of the NMR log using conventional logs as input. Algorithms based on fuzzy logic (FL), artificial neural networks (ANN) and genetic glgorithm (GA) were used to estimate the free fluid porosity and SDR permeability (Schlumberger permeability) logs starting from 5 conventional logs. Firstly, FL and ANN were applied individually to form independent intelligent systems. Later, weighting factors were assigned to each system by a average method and by GA, which was used to optimize the contribution of each individual algorithm. The methodology was applied to a dataset from Campos Basin formed by two wells. The well known P1 was used for learning and validation of intelligent systems, while the well P2 served to evaluate the extrapolation of the learning. The ANN system performed better than FL one, but this last was essential to the successful results of average (AVE) and GA systems. However, each system achieved significant approaches of the porosity and permeability curves, confirming the usefulness and reliability of the methodology.

\section{Introdução}

A descoberta de uma jazida de petróleo envolve dispendioso estudo e análise de dados geofísicos e geológicos das bacias sedimentares. Com o prognóstico do comportamento das diversas formações geológicas no subsolo e a identificação de uma área favorável à acumulação de petróleo, indica-se o local mais propício para executar a perfuração do poço. Os métodos geofísicos podem ser aplicados para determinar as distribuições das propriedades físicas em profundidade. Se corretamente aplicado, o levantamento geofísico é capaz de aperfeiçoar os programas de exploração de petróleo pela minimização das perfurações requeridas e pela maximização da taxa de cobertura da área (Kearey et al., 2002).
A perfilagem geofísica de poços, por outro lado, é parte integrante da avaliação de formação e fornece uma vasta fonte de dados relacionados à periferia do poço. Estes dados são utilizados em conjunto com dados de fluidos e testemunhos para determinar, de forma direta ou indireta, a profundidade do reservatório, espessura, porosidade, permeabilidade, litologia, saturação de hidrocarbonetos, etc. Estas informações são importantes para a perfuração, completação, operação dos poços, exploração geológica e geofísica, análise econômica e desenvolvimento de modelos de reservatórios para a produção eficiente. As medições dos parâmetros geofísicos dos poços envolvem sondas que percorrem os poços, meios de transmissão de dados para a superfície, equipamentos de registro e sistemas de processamento. As propriedades físicas são medidas e registradas em função da profundidade para obter perfis de poços, que, em seguida, são interpretados (Timur, 1982). Dentre os métodos de perfilagem geofísica de poços, destaca-se o perfil de NMR, que, segundo Coates et al. (1999), proporciona informações sobre a porosidade total, independente do tipo de rocha, saturação de água, gás e óleo, independente de outros registros, viscosidade do óleo e estimativa da permeabilidade. Além dessas medidas, Labani et al. (2010) citam ainda a porosidade de fluido livre, porosidade efetiva e o fluido adsorvido nas argilas. Portanto, esta ferramenta é capaz de prever os parâmetros petrofísicos das formações de maneira mais efetiva que as ferramentas de perfilagem convencionais, permitindo uma melhor interpretação e conduzindo a resultados mais precisos (Silva, 2010).

Porém, a técnica de NMR requer altos custos de medição, não sendo, por essa razão, obtido em todos os poços de um campo. Essa situação torna a construção de novos e avançados modelos computacionais, que sejam capazes de sintetizar os parâmetros do perfil de NMR de forma mais precisa e conveniente, de considerável importância para os estudos de caracterização de reservatórios e redução de custos (Labani et al., 2010). Na evolução dos modelos computacionais, as técnicas de inteligência artificial têm adquirido resultados relevantes na síntese do perfil NMR utilizando perfis convencionais (Nascimento \& Yoneyama, 2004; Zhou et al., 1993). Este trabalho consiste no emprego de um grupo de inteligências artificiais, tais como as ANN, FL e GA, que, atuando em conjunto, através do software interativo MATLAB (MATWORKS, 2011), sintetizará o perfil NMR avaliando sua aplicabilidade posteriormente.

\section{Metodologia}

Para realizar nosso estudo, os dados de dois poços (P1 e P2) da Bacia de Campos (Figura 1) foram utilizados. Esses poços atravessam formações pertencentes a um ambiente antigo de uma plataforma carbonática. $O$ limite 
sul da Bacia de Campos faz fronteira com a Bacia de Santos no Alto de Cabo Frio e o limite norte faz fronteira com a Bacia do Espírito Santo no Alto de Vitória.

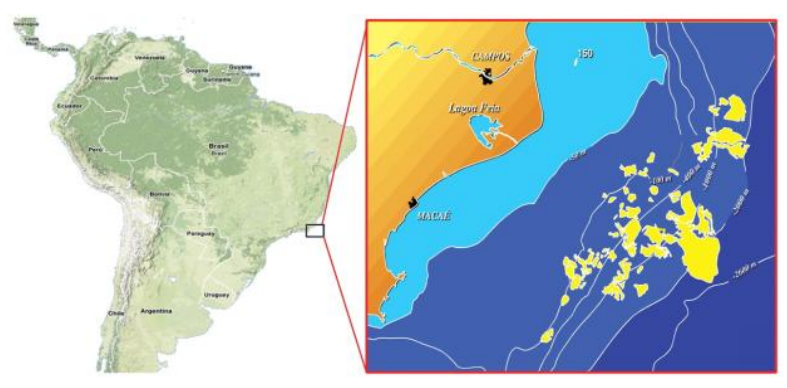

Figura 1. Localização da Bacia de Campos (modificado de Matos et al., 2008).

Franco et al. (2011) realizaram descrições sedimentológicas a partir de amostragem aleatória de plugs retirados de testemunhos da região do reservatório e adjacências. Com isso, constataram que a região está localizada numa formação carbonática com grande variação litológica. $\mathrm{Na}$ Figura 2, mostra-se o modelo deposicional esquemático sugerido para o contexto geológico da área. Ao longo da costa das Bacias de Campos e Santos, a plataforma carbonática teve sua evoluçao sedimentar condicionada por estruturas da seção pré-albiana (Figura 3). A carga sedimentar, inclinação do substrato e reativações de falhamentos influenciaram a movimentação dos evaporitos, controlando a geometria e distribuição das fácies litológicas.

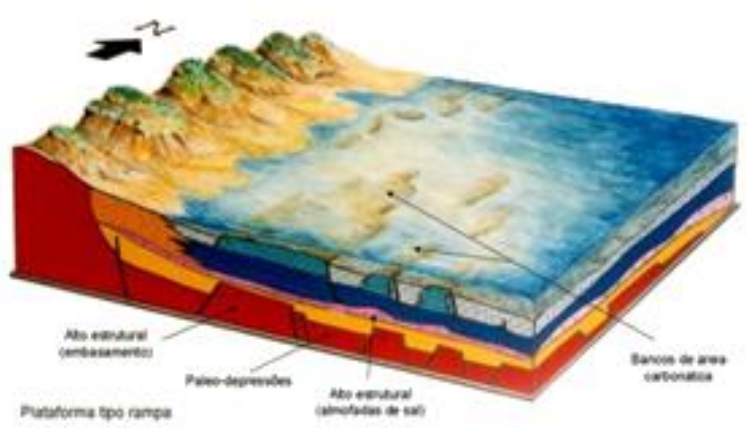

Figura 2. Modelo deposicional esquemático de uma rampa carbonática (modificado de Matos et al., 2008).

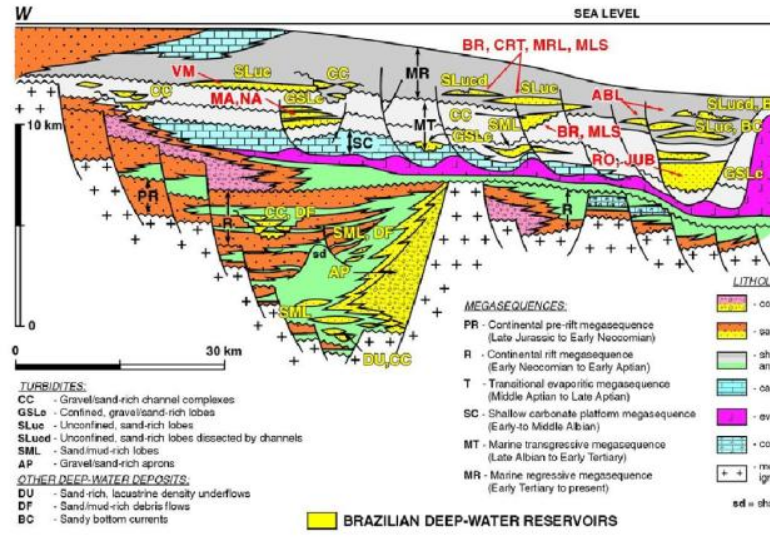

Figura 3. Reservatórios da Bacia de Campos (modificado de Bhrun et al.,2003 ).

Através das descrições sedimentológicas citadas anteriormente, também foi possível identificar as quatro litofácies principais do intervalo de profundidade utilizado neste trabalho: packstone, grainstone, grainstone cimentado e dolomita. A dolomita encontra-se abaixo da região de grainstone cimentado (Figura 4).

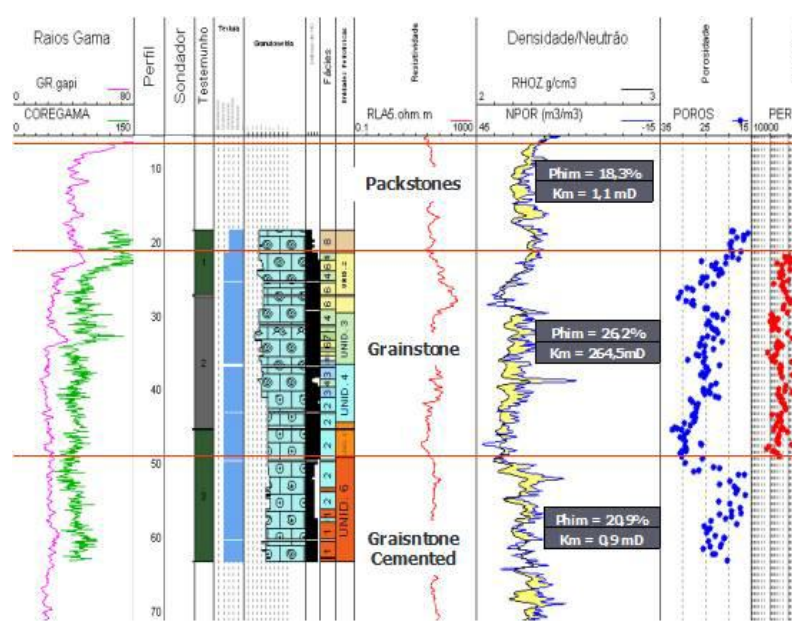

Figura 4. Litofácies da região do reservatório (modificado de Franco et al., 2011).

Os sistemas inteligentes foram assim alimentados com os perfis geofísicos convencionais desses 2 poços, destacados a seguir: 5 perfis convencionais (raios gama (GR), resistividade laterolog (RLA1), densidade (RHOZ), porosidade neutrônica (NPOR), sônico (DTCO)), perfil de porosidade de fluido livre NMR (CMFF) e perfil de permeabildade SDR NMR (KSDR). Os perfis do poço P1 são mostradas na Figura 5. No lado esquerdo da figura estão os perfis convencionais utilizados como entrada nos algoritmos inteligentes, e, no lado direito, os perfis de porosidade e permeabilidade, correspondendo aos perfis alvo (CMFF e KSDR). Na Figura 6, encontram-se as curvas dos perfis do poço P2. Da mesma forma, no lado 
esquerdo da figura estão os perfis de entrada, perfis convencionais, e, no lado direito, os perfis alvo, porosidade e permeabilidade.

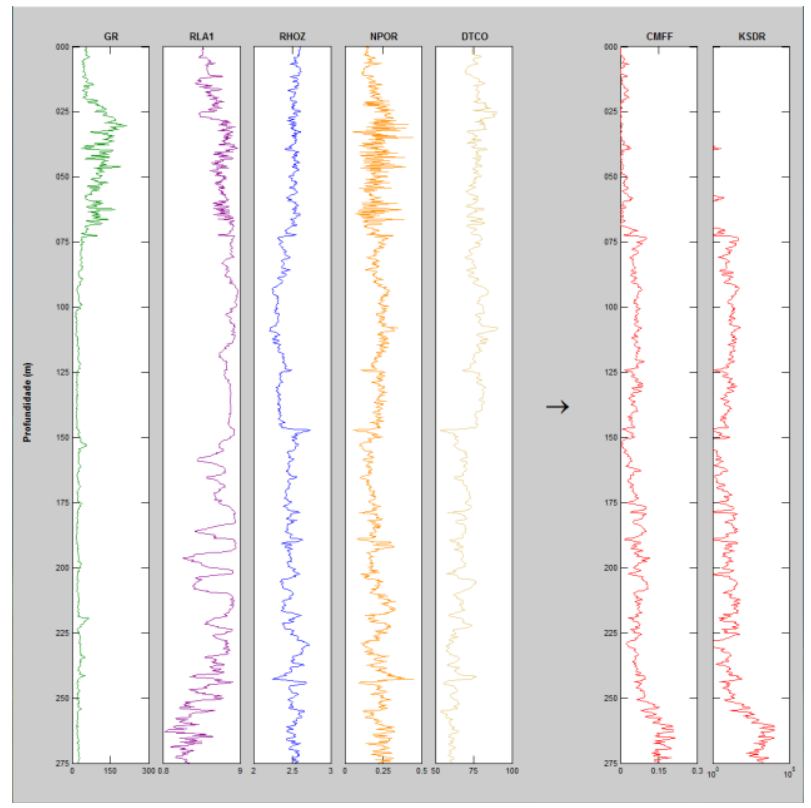

Figura 5. Perfis convencionais, porosidade e permeabilidade NMR do poço P1.

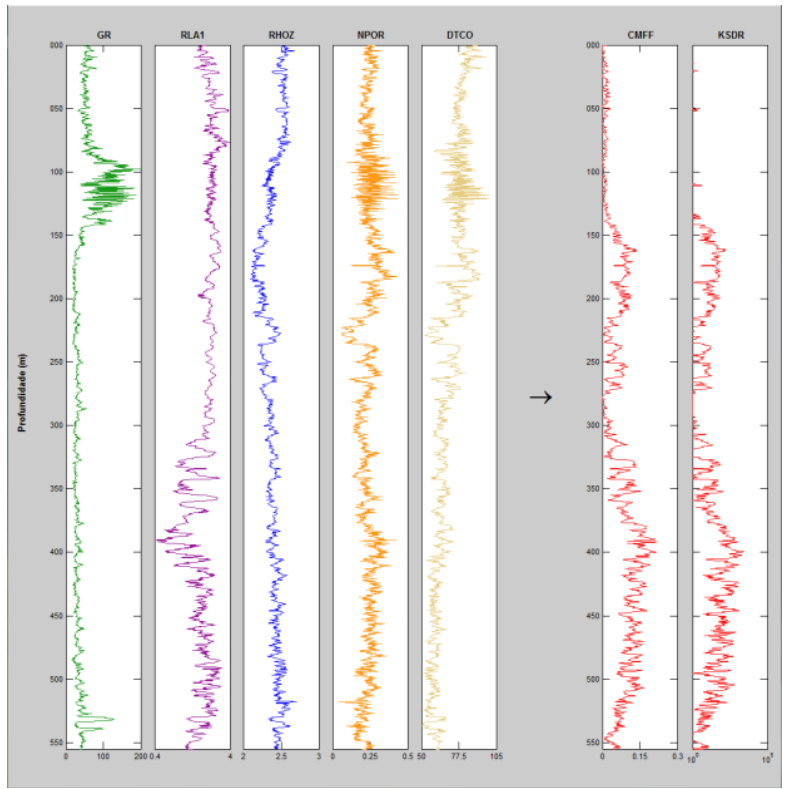

Figura 6. Perfis convencionais, porosidade e permeabilidade NMR do poço P2.

\section{Discussão}

A seguir, nas Figuras 7, 8, 9 e 10, mostram-se os perfis resultantes da validação das estimativas para $\mathrm{FL}, \mathrm{ANN}$, AVE e GA para o poço P1. Em vermelho, estão as curvas de porosidade de fluido livre (CMFF) e permeabilidade SDR (KSDR), e, em azul, estão as curvas geradas pelos algoritmos de inteligência artificial a partir dos perfis convencionais do mesmo poço.

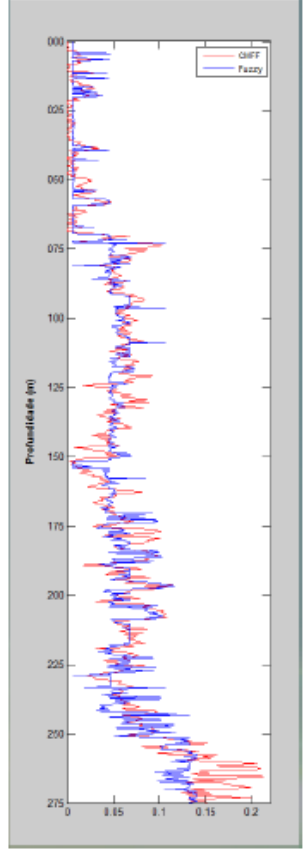

(a) Porosidade.

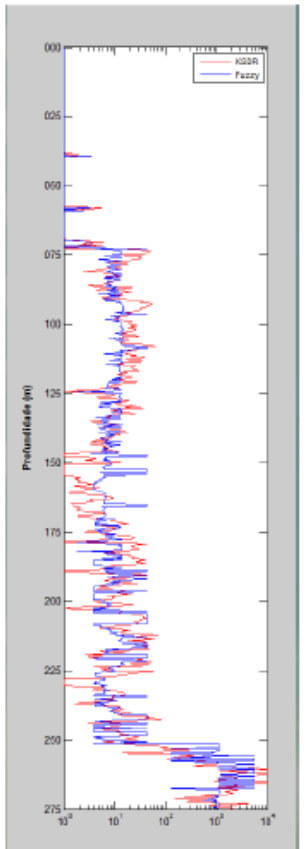

(b) Permeabilidade.
Figura 8. Previsão dos perfis de RMN do poço P1 através do sistema FL.

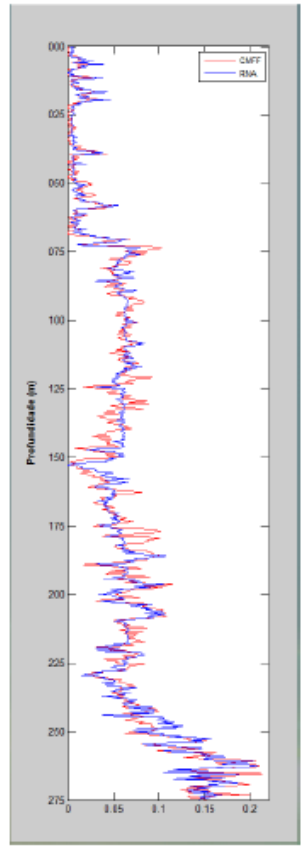

(a) Porosidade.

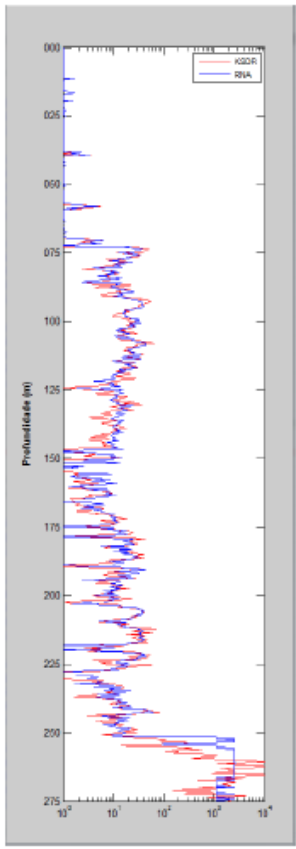

(b) Permeabilidade.
Figura 7. Previsão dos perfis de RMN do poço P1 através do sistema ANN. 


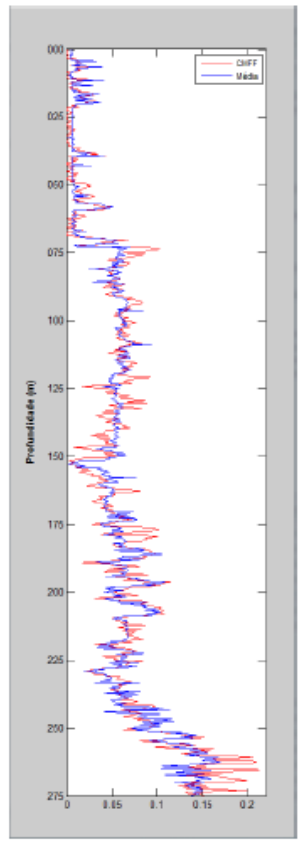

(a) Porosidade.

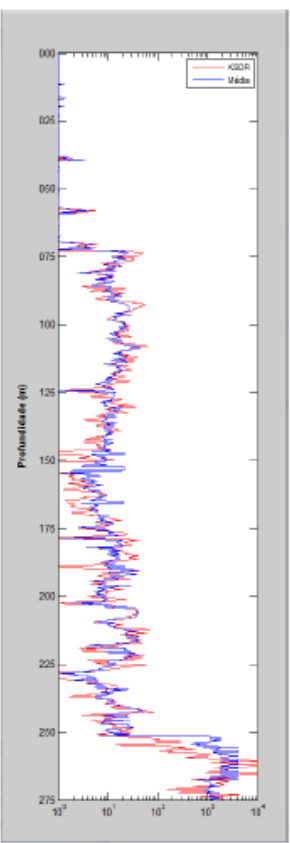

(b) Permeabilidade.

Figura 9. Previsão dos perfis de RMN do poço P1 através do sistema AVE.

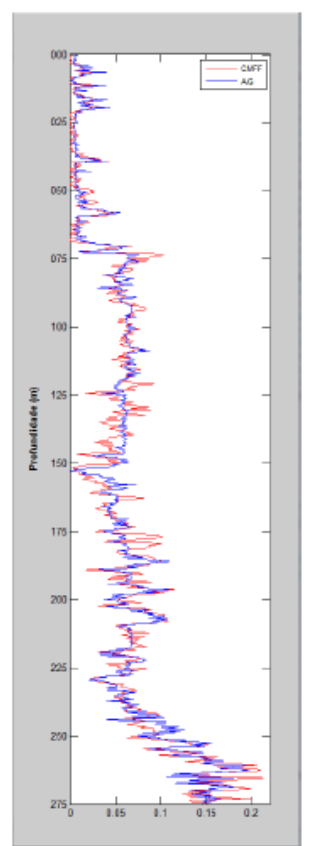

(a) Porosidade

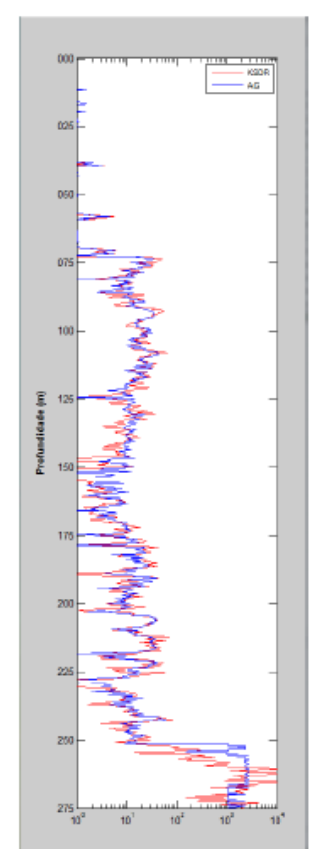

(b) Permeabilidade.
Figura 10. Previsão dos perfis de RMN do poço P1 através do sistema GA.
$\mathrm{Na}$ Tabela 1, são apresentados os erros dos perfis de porosidade simulados, bem como a classificação de cada sistema inteligente de acordo com a melhor aproximação e menor erro com a curva alvo. $\mathrm{Na}$ Tabela 2, por outro lado, são apresentados os erros dos perfis de permeabilidade simulados e a classificação de cada sistema inteligente de acordo com a melhores resultados de previsão.

Tabela 1. Comparação entre os erros das simulações de porosidade, em MSE é o erro quadrático médio.

\begin{tabular}{|c|c|c|c|c|}
\hline \multirow{2}{*}{ Sistema Inteligente } & \multicolumn{2}{|c|}{ Validação (Poço P1) } & \multicolumn{2}{c|}{ Previsão (Poço P2) } \\
\cline { 2 - 5 } & $M S E(\mathrm{pu})^{2}$ & Classificação & $M S E(\mathrm{pu})^{2}$ & Classificação \\
\hline \hline Fuzzy & 0,00035048 & 4 & 0,00182876 & 4 \\
\hline Redes Neurais & 0,00017897 & 2 & 0,00126872 & 3 \\
\hline Média & 0,00020668 & 3 & 0,00123483 & 1 \\
\hline Algoritmos Genéticos & 0,00017390 & 1 & 0,00124656 & 2 \\
\hline
\end{tabular}

Tabela 2. Comparação entre os erros das simulações de permeabilidade, em MSE é o erro quadrático médio.

\begin{tabular}{|c|c|c|c|c|}
\hline \multirow{2}{*}{ Sistema Inteligente } & \multicolumn{2}{|c|}{ Validação (Poço P1) } & \multicolumn{2}{c|}{ Previsão (Poço P2) } \\
\cline { 2 - 5 } & $M S E(m D)^{2}$ & Classificação & $M S E(m D)^{2}$ & Classificação \\
\hline \hline Fuzzy & 715519,9231 & 4 & 2999840 & 4 \\
\hline Redes Neurais & 488169,7611 & 2 & 254018 & 1 \\
\hline Média & 528990,8120 & 3 & 1108540 & 3 \\
\hline Algoritmos Genéticos & 485011,4734 & 1 & 300658 & 2 \\
\hline
\end{tabular}

\section{Conclusões}

A perfilagem de NMR fornece informações valiosas para a avaliação petrofísica dos reservatórios de hidrocarbonetos. Porém, apesar de sua vantagens, a técnica requer altos custos de medição e por isso não é realizado em todos os poços perfurados num reservatório. Portanto, a construção de novos e avançados modelos computacionais capazes de sintetizar os parâmetros do perfil de NMR é de considerável importância para a redução de custos na fase de caracterização de reservatórios. Técnicas de inteligência artificial para a estimativa de diversos parâmetros em diferentes disciplinas científicas são utilizadas há décadas, e muitos trabalhos já foram publicados com aplicabilidade considerável. Nesse estudo, essas técnicas foram utilizadas individualmente e em conjunto para estimar os perfis de porosidade de fluido livre e permeabilidade SDR do perfil de NMR, usando como entrada 5 perfis geofísicos convencionais da Bacia de Caampos, a saber: GR, RLA1, RHOZ, NPOR e DTCO. O poço denominado P1 foi utilizado para 0 aprendizado e validação dos sistemas inteligentes, e o poço P2 para avaliar a extrapolação do aprendizado. Além dos perfis de poço, foram obtidas descrições sedimentológicas de plugs de testemunho, o que facilitou a compreensão da litologia atravessada pelos poços. Assim, foram construídos 4 sistemas inteligentes, sendo que $F L$ e ANN formaram sistemas independentes, enquanto que os sistemas AVE e GA buscaram aperfeiçoar os resultados das anteriores. $O$ processo de 
aprendizagem da ANN, com a aplicação do algoritmo de retropropagação (supervisão forte), se destacou em relação ao aprendizado da FL. Afinal, enquanto o supervisor necessita de dias para fazer os ajustes das funções de pertinência do sistema FL, a ANN executa a mesma tarefa em algumas horas. A ANN utilizada possuiu supervisão forte, em que não há controle sobre o aprendizado da inteligência, assim que, quanto mais treinada era, melhores eram os resultados do processo de validação. Porém, quanto mais a rede se adapta a um problema específico, mais perde a capacidade de abstrair-se em novos problemas. Com isso, longos treinamentos com redes muito inteligentes resultaram em extrapolações com alto valor de MSE (Mean Square Error - erro quadrático médio). A solução foi utilizar uma quantidade menor de épocas e poucos neurônios, para que a ANN pudesse aprender somente o essencial no poço P1 e extrapolar esse aprendizado na estimativa do poço P2. No caso da FL, quanto mais o sistema é ajustado, melhores resultados de extrapolação são obtidos. Afinal, comparando com uma ANN, a FL seria uma rede com supervisão muito forte, o que limitaria o aprendizado da inteligência à somente o necessário através da maior liberdade do supervisor. Contanto, é necessário considerar o custo de supervisionar o processo de aprendizado, como citado anteriormente. Entre vantagens e desvantagens, o sistema ANN obteve melhor desempenho comparado ao sistema FL, porém, este último foi fundamental para a criação dos bons resultados dos sistemas AVE (média) e GA. No processo de validação, a mesma sequência classificatória dos resultados das aproximações da porosidade e permeabilidade deixa claro que o sistema GA cumpriu sua tarefa de otimização. Forneceu pesos ótimos para o sistemas ANN e FL, destacando-se, em primeiro lugar. Por outro lado, o sistema AVE apenas comprovou que a estimativa do sistema ANN foi superior à estimativa do sistema FL. No processo de extrapolação da porosidade, os pesos do sistema GA, otimizados no processo de validação, não conseguiram superar 0 resultado adquirido pelos pesos iguais do sistema AVE. Já no processo de extrapolação da permeabilidade, como o sistema ANN foi mais de 10 vezes superior ao sistema $\mathrm{FL}$, quanto que os sistemas AVE e de GA não conseguiram melhorar o resultado da ANN. Contudo, é importante destacar que o MSE não é a forma ideal de se avaliar o erro entre curvas logarítmicas. Finalmente, o presente trabalho comprovou a eficiência da utilização de sistemas inteligentes, trabalhando tanto individualmente, quanto em conjunto, na estimativa de parâmetros petrofísicos do perfil NMR. Mesmo que os sistemas inteligentes tenham obtido posições variadas na classificação dos resultados, cada um deles alcançou estimativas razoáveis das curvas de porosidade e permeabilidade. Dessa forma, a extrapolação de perfis para outros poços através do aprendizado das técnicas de inteligência artificial é mais uma ferramenta à disposição para a caracterização de reservatórios fornecendo resultados úteis e confiáveis.

\begin{abstract}
Agradecimentos
À PETROBRAS por ceder os dados de reservatório da Bacia de Campos para a realização deste estudo.
\end{abstract}

\section{Referências}

Bruhn, C.; Gomes, J.; Lucchese. C. \& Johann, P. 2003. Campos Basin: Reservoir Characterization and Management - Historical Overview and Future Challenges. Offshore Technology Conference, p. 15220, $14 \mathrm{pp}$.

Coates, G.; Xiao, L.; Prammer, M. 1999. NMR Logging: principles \& applications. Houston: Halliburton Energy Services, $234 \mathrm{p}$.

Franco, N. P.; Schuab, F.; Nocchl, G. 2011. Estudo para determinação dos cortes de t2 através da integração entre a distribuição de t2 do perfil de ressonância magnética com a distribuição de garganta de poros do ensaio de pressão capilar por injeção de mercúrio. Relatório do Projeto Análise de dados de reservatórios. Convênio Petrobras/LENEP-UENF/Fundação BioRio, 76 pp.

Labani, M..; Kadkhodaie-llkhchi, A.; Salahshoor, K. 2010. Estimation of NMR log parameters from conventional well log data using a committee machine with intelligent systems: A case study from the iranian part of the south pars gas field, Persian Gulf Basin. Journal of Petroleum Science and Engineering, v. 72, p. 175-185.

Kearey, P.; Brooks, M. \& Hill, I. 2002. An Introduction to Geophysical Exploration. 33a. ed. Oxford: Blackwell Science, $262 \mathrm{p}$.

MATHWORKS, T. MATLAB User's Guide 2011. Fuzzy logic, Neural Network, Genetic Algorithm and direct search toolboxes. [S.I.].

Nascimento, C. \& Yoneyama, T. 2004. Inteligência Artificial em Controle e Automação. São Paulo: Edgard Blücher, 218 p.

Serra, O. 1998. Fundamentals of Well-log Interpretation. Amsterdam: Elsevier, $422 \mathrm{p}$.

Silva, C.. 2010. Simulação do perfil de Ressonância Magnética Nuclear (RMN) utilizando perfis geofísicos de poços convencionais através da lógica fuzzy. Dissertação de Mestrado, LENEP/UENF, Macaé - RJ, 125 pp.

Timur, A. 1992. Advances in well logging. Journal of Petroleum Technology, v. 1, p. 16 - 25.

Zhou, C.; Wu, X.; Cheng, J. 1993. Determining reservoir properties in reservoir studies using a fuzzy neural network. SPE Annual Technical Conference and Exhibition, v. 1, p. 3-6. 